\title{
Systèmes De Mesure De La Performance (SMP) Dans Les PME Marocaines: Les Résultats D’une Recherche Exploratoire
}

\author{
Salwa Bahyaoui, (Enseignante-chercheur) \\ Faculté des Sciences Juridiques, Economiques et Sociales - Agdal- \\ Université Mohammed V de Rabat, Maroc
}

doi: 10.19044/esj.2017.v13n10p149 URL:http://dx.doi.org/10.19044/esj.2017.v13n10p149

\begin{abstract}
Despite having experienced profusion since the mid-90s, research on Performance Measurement Systems (PMS) has remained focused on large companies. Interest in the study of such systems in the context of SMEs is not only relatively recent, but occurs mainly in developed countries. This triggered our interest in understanding performance measures in a developing country, namely, Morocco. To do this, an exploratory empirical study was conducted among a sample of Moroccan SMEs. The results, while confirming some specific characteristics of SMEs, help qualify some aspects including those related to the lack of strategic vision and the use of quantitative measures rather than qualitative ones. This paves the way for further research in this area.
\end{abstract}

Keywords: SMEs, Performance Measurement Systems (PMS), Contingency factors, Morocco, Logistic regression

\section{Résumé}

La recherche sur les SMP, bien qu'ayant connu un foisonnement depuis la moitié des années 90s, est restée focalisée sur les grandes entreprises. L'intérêt pour l'étude desdits systèmes dans le contexte des PME, est non seulement relativement récent, mais se manifeste principalement dans les pays développés. Ce qui a suscité notre intérêt à appréhender la problématique de la mesure de la performance dans un pays en développement, en l'occurrence, le Maroc. Pour ce faire, une étude empirique exploratoire a été menée auprès d'un échantillon de PME marocaines. Les résultats obtenus, tout en confirmant certaines spécificités des PME, permettent de nuancer quelques aspects notamment ceux liés à l'absence de vision stratégique et à l'usage de mesures plutôt quantitatives 
que qualitatives. Ce qui ouvre la voie à des recherches plus approfondies en la matière.

Mots-clés: PME, Systèmes de Mesure de la Performance SMP, Facteurs de contingence, Maroc, Régression logistique binaire

\section{Introduction}

Depuis le début des années 90s, plusieurs chercheurs (Kaplan et Norton, 1992, Russell, 1992 ; Bititci, 1995, Lebas, 1995) s’accordent à souligner l'intérêt pour les entreprises à se doter d'un SMP en vue d’améliorer leurs pratiques managériales.

Ces années marquent également le passage des modèles « traditionnels » de mesure de la performance, basés fondamentalement sur des indicateurs financiers, vers une approche plus holistique des SMP. En effet, depuis la publication des travaux de Kaplan et Norton (1992, 1996) sur les tableaux de bord stratégiques, plusieurs chercheurs se sont attelés à développer des modèles dits « intégrés » ou « équilibrés ». En plus d’être fondés sur des indicateurs financiers et non financiers, ces modèles tentent d’intégrer les objectifs stratégiques de l'entreprise ainsi que les intérêts des parties prenantes (stakeholders) afin de mieux traduire la performance de l'entreprise pour mieux la piloter (Bititci et al., 1997, Ghalayini et al., 1997, Neely et al., 2000, Neely et al., 2001).

Toutefois, la quasi-totalité des modèles proposés ainsi que les études empiriques sur le sujet se sont particulièrement intéressés aux grandes entreprises dans les pays développés. Très peu de recherches ont été menées dans un contexte de PME (Bergeron, 2000, Garengo et Bititci, 2007). En outre, celles-ci restent limitées au cas des PME dans des pays développés notamment en Australie et au Royaume-Uni. Quant aux pays émergents, l'exploration des SMP dans les entreprises en général et dans les PME en particulier, reste très sporadique et relativement récente (Shahbaz et al., 2014, Akpabot et Khan, 2015).

Or dans un pays comme le Maroc, où le tissu économique est constitué majoritairement de PME, la prospérité de celles-ci conditionne le développement économique du pays. Cette prospérité est elle-même dépendante, partiellement, des outils de gestion et de pilotage de la performance (Bergeron, 2000).

En outre, les recherches récentes relèvent le gap important entre les modèles théoriques de SMP et leur mise en pratique en raison, notamment, de leur inadaptation aux changements de l'environnement et aux spécificités des organisations (Bititci et al., 2012, Melnyk et al., 2014).

Ainsi, l’objectif principal de cette contribution est de saisir la réalité de la mesure de la performance dans un contexte de PME évoluant dans un 
pays en développement, en tentant de répondre principalement aux questions de recherche suivantes :

- Comment les PMEs marocaines mesurent-elles leur performance ?

- Existent-ils des facteurs de contingence qui influencent la structure et l'usage des SMP dans les PME ?

Pour ce faire, cet article sera divisé en trois grandes parties :

- une première partie récapitulant les principales études ayant porté sur les systèmes de mesure de la performance dans les PME ;

- la deuxième partie exposera la méthodologie adoptée pour répondre à nos objectifs de recherche ;

- et enfin, la troisième partie sera consacrée à l'analyse des résultats de l'étude empirique menée auprès d'un échantillon de PME.

\section{Cadrage théorique : PME et systèmes de mesure de la performance}

La définition des PME est loin de faire l'unanimité, encore moins celle des systèmes de mesure de la performance $\mathrm{y}$ afférent. Ce qui nous amène à préciser d'abord la définition de la PME qui sera adoptée dans notre recherche (1.1), avant de se pencher sur les SMP spécifiques au contexte des PME (1.2).

\section{Définitions des PME : diversité et spécificités}

Beaucoup de chercheurs, ont relevé très tôt, la difficulté de définir et de délimiter avec précision le champ d’étude des PME (White et al., 1982 ; D’Amboise, 1996 ; Torrès, 1999). Cette difficulté est inhérente, d'une part à la diversité des PME qui constituent des organisations hétérogènes; et d'autre part, à leurs spécificités les différenciant des grandes entreprises dont elles ne peuvent être de simples modèles réduits.

En effet, l'extrême hétérogénéité des PME pose le problème de délimitation du champ d'étude pour la plupart des recherches dans ce domaine (Julien, 1998, Guilhon, 1998). Torrès (1999) affirme, dans ce sens, que : « la PME n’existe pas, la diversité des cas est telle qu'il convient mieux de dire les PME. Le monde des PME est foisonnant de variété...... Cette diversité explique la grande difficulté que les chercheurs ont éprouvé lorsqu’il s'est agi de théoriser le phénomène du Small is beautiful ». Cette situation entrave la comparaison des résultats et des conclusions des études dans ce domaine, surtout lorsqu'elles sont menées dans des contextes et des environnements différents.

En outre, et comme l’ont bien souligné Welsh et White (1981) : «A small business is not a little big business». Ainsi, de nombreux chercheurs, reconnaissant la spécificité des PME, se sont attelés à identifier des critères de leur classification en des catégories plus ou moins homogènes (Julien et 
Marchesnay, 1996, Torrès, 2008). Sommairement, deux familles de classement coexistent :

- les typologies quantitatives faisant référence aux aspects représentatifs de la taille de l'entreprise. Les indicateurs les plus couramment utilisés sont quantitatifs comme l'effectif, le chiffre d'affaires ou encore la part de marché ;

les typologies qualitatives ou multicritères qui mettent l'accent sur les spécificités managériales et organisationnelles des PME telles que l'omniprésence de la personne du propriétaire-dirigeant dans la gestion, la stratégie implicite et peu formalisée, un système d’information simple ainsi que le circuit décisionnel orienté vers le court terme.

Toutefois, et en dépit des différentes approches et tentatives de définition des PME, c’est le critère «taille », mesuré par l'effectif employé, qui reste le plus souvent utilisé (Allali, 2003). En effet, celui-ci a l'avantage d'être un dénominateur commun, relativement facile à recueillir et simple à utiliser malgré son caractère réducteur (OCDE, 2004, Oriot et Bergeron, 2012).

L’Union européenne et plusieurs pays de l’OCDE ainsi que des pays en transition et en développement fixent la limite supérieure à 200 ou 250 personnes $^{1}$. Peu de pays dérogent à la règle : le Japon (300 salariés), les États-Unis et le Canada (500 salariés). D’autres critères sont souvent pris en compte en plus des effectifs. Il s'agit notamment du chiffre d'affaires (CA) et/ou du total bilan (TB).

Au Maroc, plusieurs définitions de la PME coexistent. La « version officielle » donnée par la charte de la $\mathrm{PME}^{2}$ retient trois critères pour définir une PME à savoir la gérance, la propriété du capital et la taille. Néanmoins, aucune distinction n'est faite entre les TPE (Très Petites Entreprises), les PE (Petites Entreprises) et les EM (Entreprises Moyennes).

D’autres institutions adoptent des définitions différentes. Il s’agit notamment de Bank Al-Maghrib ${ }^{3}$ et de l'ANPME ${ }^{4}$ (Agence Nationale pour la Promotion de la PME). Celles-ci se basent notamment sur le CA pour faire la distinction entre les TPE, les PME et les grandes entreprises. Toutefois, les seuils de catégorisation retenus sont légèrement différents.

Le tableau qui suit récapitule les principales définitions aux niveaux international et national.

1 «Chapitre 1: Caractéristiques et importance des PME », Revue de l'OCDE sur le développement 2/2004 ( $\mathrm{n}^{\circ}$ 5), p. 37-46.

${ }^{2}$ Charte promulguée en 2002 par la loi n53-00 au Bulletin officiel 5036 du 5/9/2002.

${ }^{3}$ Bank Al-Maghrib est la banque centrale du Maroc.

${ }^{4}$ Depuis 2015, l'Agence a adopté une nouvelle identité visuelle : « Maroc PME® » et un nouveau slogan : «entrepreneuriat - croissance - compétitivité » pour accompagner sa nouvelle mission stratégique. 
TABLEAU 1: Tableau récapitulatif des critères et des définitions des PME

\begin{tabular}{ccccc}
\hline $\begin{array}{c}\text { Organismes } \\
\text { ou pays }\end{array}$ & $\begin{array}{c}\text { Critère(s) } \\
\text { retenu(s) }\end{array}$ & $\begin{array}{c}\text { Micro-entreprise ou } \\
\text { Très Petite } \\
\text { Entreprise (TPE) }\end{array}$ & $\begin{array}{c}\text { Petite Entreprise } \\
\text { (PE) }\end{array}$ & $\begin{array}{c}\text { Entreprise } \\
\text { Moyenne (EM) }\end{array}$ \\
\hline OCDE & Effectif & $<10$ salariés & 10 à 49 salariés & 50 à 249 salariés \\
\hline UE & $\begin{array}{c}\text { Effectif et } \\
\text { CA ou total } \\
\text { Bilan }\end{array}$ & $\begin{array}{c}\text { Effectif }<10 \\
\text { et } \\
\text { CA ou TB } \leq 2 \text { Mio } €\end{array}$ & $\begin{array}{c}\text { Effectif }<50 \\
\text { et }\end{array}$ & $\begin{array}{c}\text { CA ou TB } \leq 10 \text { Mio } € \\
\text { et CA } \leq 50 \text { Mio } € \\
\text { ou TB } \leq 43 \text { Mio } €\end{array}$ \\
\hline $\begin{array}{c}\text { Maroc } \\
\text { Charte de } \\
\text { la PME }\end{array}$ & $\begin{array}{c}\text { La gérance, } \\
\text { la propriété } \\
\text { du capital, } \\
\text { l'effectif et le } \\
\text { CA et/ou TB }\end{array}$ & $\begin{array}{c}\text { La gérance doit être assurée directement par des personnes } \\
\text { physiques ; }\end{array}$ \\
$\begin{array}{c}\text { Le capital ne peut être détenu à plus de 25\% par une entreprise ou un } \\
\text { Effectif }<200 \text { salariés et CA } \leq 75 \text { Mio MAD et/ou TB } \leq 50 \text { Mio } \\
\text { MAD. }\end{array}$ \\
\hline
\end{tabular}

La définition de la charte demeure complexe car il est très difficile, en l'absence de statistiques détaillées sur les entreprises, de pouvoir combiner les trois critères simultanément. En outre, le critère de taille est, lui-même, mesuré par deux indicateurs que sont l'effectif permanent et le chiffre d'affaires et/ou le total bilan.

Ainsi, et pour les besoins de notre recherche, nous nous sommes alignés sur la classification de l'OCDE et de l'UE basée sur l'effectif, tout en le croisant avec le CA.

\section{Les SMP en contexte de PME : revue des principales recherches}

Les SMP, comme d'ailleurs le concept de performance auquel ils sont rattachés, sont loin de constituer un corpus cohérent malgré le foisonnement de la littérature soulignant l'intérêt pour les entreprises de se doter de SMP. Ce qui s'est traduit par la prolifération des définitions.

En effet, Franco-Santos et al. (2007) ont recensé pas moins de 17 définitions des SMP sur la période allant de 1990 à 2003 ; définitions souvent divergentes en fonction de la perspective d'analyse adoptée. Cette absence de cohérence et de clarté crée, selon ces auteurs, une confusion et des problèmes de comparabilité qui constituent une entrave majeure au développement de la recherche dans le domaine.

Toutefois, malgré la diversité des définitions recensées dans la littérature, celles-ci se recoupent au moins sur deux points comme le notent Franco-Santos et al. (2012) :

- $\quad$ les composants des SMP : pour être défini comme tel, un SMP doit comprendre un ensemble de mesures financières et non financières de la performance ;

- l'usage/l'objectif des SMP : les mesures utilisées doivent permettre de rendre opérationnels les objectifs stratégiques. En d'autres termes, les 
SMP doivent être des outils de management et de pilotage de la performance et non seulement des outils de contrôle et de suivi.

En revanche, s’il existe une littérature abondante sur les SMP d'une part et sur les PME d'autre part, très peu de recherches concernent les SMP dans les PME (Anderson et al, 2001, Garengo et Bititci, 2007, Brem et al., 2008). En effet, Brem et al., (2008) ont identifié moins de 50 articles traitant des SMP dans les PME durant la décennie 1998-2008, contrastant avec les 3615 articles sur la mesure de la performance dans les grandes entreprises entre 1994 et 1996 (Neely, 1999).

Taticchi et al. (2010) notent l'intérêt relativement récent pour l'étude des SMP dans un contexte de PME qui n’a réellement démarré que vers le début des années 2000. Dans un premier temps, la recherche s’est d'abord orientée principalement vers l'adaptation et ou l'application de modèles initialement conçus pour les grandes entreprises au contexte des PME ; avant de s'orienter, dans un deuxième temps, vers la conception de SMP spécifiques aux PME. En effet, sur 14 modèles recensés entre 2000 et 2008 par Taticchi et al. (2010), seuls trois constituent des modèles intégrés pour la mesure et le management de la performance des PME. La majorité constitue notamment une transposition du BSC aux PME (Langwerden, 2015).

Brem et al. (2008) ont également noté que la majorité des articles traitant la problématique des SMP dans les PME sont basés sur une perspective " instantanée » qui ne considère que les phases de design et d'implantation des SMP, peu d'informations sont fournies quant à l'usage et à la réussite desdits SMP.

En outre, le peu d'études théoriques et empiriques menées dans un contexte de PME sont menées en Australie (Barnes, 1998, Chennell, 2000), en Finlande (Laitinen, 2002), au Royaume-Uni (Bititci et al. 2000) et au Danemark (Hvolby, et Thorstenson, 2000). Quant aux pays émergents, l'exploration des SMP dans les entreprises en général et dans les PME en particulier, reste très sporadique et relativement récente (Shahbaz et al. 2014, Akpabot et Khan 2015).

A l'issue de ces recherches, plusieurs auteurs notent que les PME sont très en retard dans l'introduction de SMP (Garengo et al., 2005, Brem et al., 2008, Nudurupati, 2011). Plusieurs raisons ont été invoquées dans la littérature dont nous présenterons les plus importantes :

a. La difficulté à impliquer les PME dans un projet de mesure de la performance : Brem et al. (2008) insistent sur la préparation des conditions de réussite à l'implantation d'un SMP notamment la formulation technique de la stratégie, un système d'information adéquat et des ressources suffisantes. Ce qui risque de dissuader la mise en place d'un tel SMP ;

b. Soit les PME n'utilisent aucun SMP, soit lorsqu'il existe, celui-ci n’est pas correctement utilisé, ou encore se limite à transposer des modèles 
initialement conçus pour les grandes entreprises sans considération des spécificités des PME (Garengo et al., 2005). Certains auteurs en ont conclu qu'un SMP adapté aux PME doit utiliser les ressources de façon efficiente et produire des résultats aussi bien à court terme qu'à long terme (Meyer, 2007);

c. Lorsqu'ils existent, ces SMP sont rarement « intégrés » selon une approche holistique de la performance: les PME focalisent généralement leur attention sur les aspects opérationnels et financiers de la performance. Les SMP sont généralement vus comme une source de bureaucratie et un obstacle à la flexibilité (Hvolby et Thorstensen, 2000) ;

d. L'approche des PME pour la mesure de la performance est souvent « informelle » : elle n’est ni planifiée, ni basée sur un modèle prédéfini mais le plus souvent adoptée à l'occasion de la résolution d'un problème spécifique. Ce qui rend la tâche d’implémentation de SMP formels encore plus difficile (Brem et al., 2008) ;

e. Les PME ont des ressources limitées aussi bien pour la collecte que pour l'analyse des données, ce qui se traduit par une ambiguïté des objectifs mesurés. En effet, la principale contrainte chez les PME qui affecte non seulement le SMP mais aussi toutes leurs activités est le manque de ressources aussi bien humaines, financières que temporelles (Hudson et al., 2001, Garengo et al., 2005). En outre, les PME manquent généralement d'infrastructure en nouvelles technologies de l'information (NTI) et de bases de données solides et fiables ;

f. Le style de management du propriétaire-dirigeant: beaucoup d'auteurs s'accordent à dire que la personne du propriétaire-dirigeant a une influence significative sur les choix stratégiques de l'entreprise, qui sont généralement définis en fonction de leurs désirs et leurs vécus et non pas sur la base d'une analyse rationnelle (Bergeron, 2000, St-Pierre et Cadieux, 2011, Oriot et Bergeron, 2012) ;

g. Un autre problème soulevé par Hudson (2001) et qui n’est pas particulièrement spécifique aux PME c'est que la mesure de la performance conduit à produire une pléthore d'informations qui non seulement est complexe à analyser mais devient vite désuète et inexploitable. Ainsi, les PME seraient réticentes à mettre en place des modèles complexes dont elles n'en ont pas vraiment besoin. Un SMP clair et simple serait plus flexible et adapté aux changements de l'environnement des PME (Meyer, 2002, Garengo et al., 2005).

Ainsi, notre objectif sera, d'une part, de confronter la réalité de la mesure de la performance dans les PME marocaines avec les résultats des principales recherches dans le domaine ; et d'autre part de déceler, le cas échéant, les facteurs de contingence liés à la mesure de la performance dans les PME. 


\section{Méthodologie de recherche}

Comme nos objectifs de recherche visent à explorer des réalités diverses, multidimensionnelles et holistiques (les systèmes de mesure de la performance) construites à partir d'interactions sociales subjectives (PME dans le contexte marocain), nous nous sommes référés au point de vue épistémologique socioconstructiviste (Vygotsky, 1978).

Pour répondre à ces objectifs, nous avons adopté une double approche :

- $\quad$ dans un premier temps, et compte tenu du volet exploratoire de notre étude, nous avons privilégié une approche qualitative comme le suggère la littérature (Miles et Huberman, 2003, Strauss et Corbin, 2004) ; et ce en vue de saisir la nature des indicateurs et des SMP utilisés par les propriétairesdirigeants des PME enquêtées ;

- dans un deuxième temps, et pour étudier les éventuels facteurs de contingence qui influenceraient la structure et l'usage desdits SMP, nous nous sommes basés sur une analyse quantitative des données recueillies.

\section{Recueil des données}

Les données utilisées sont extraites de la base fournie par l'ANPME « Maroc PME » relative à 334 TPE et 515 PME au sens de la catégorisation retenue par l'Agence. Cette base de données a été construite à partir d'un questionnaire comprenant un ensemble de variables dont nous n’avons été autorisés à exploiter que la partie directement liée à nos objectifs de recherche et qui comprend :

- $\quad$ trois variables quantitatives : capital social, chiffre d'affaires annuel moyen, effectifs permanents employés ;

- $\quad$ deux variables qualitatives : les objectifs poursuivis pour améliorer la performance et les indicateurs de mesure fréquemment utilisés. Ce sont deux questions ouvertes auxquelles ont répondu soit les propriétaires-dirigeants eux-mêmes, soit les gérants des PME ;

- $\quad$ trois variables catégorielles : secteurs d'activité, régions et âge de la PME.

- $\quad$ sept variables dichotomiques: besoin d'un ERP, besoin pour l'alignement stratégique, besoin d'accompagnement du dirigeant, accès facile au financement, ressources humaines compétentes, usage du SMP pour le pilotage et vision stratégique claire. 
TABLEAU 2: Définition des principales variables

\begin{tabular}{|c|c|c|}
\hline Variables & Définition & Nature et modalités \\
\hline SIZE & Effectifs permanents & Variable quantitative discrète \\
\hline AGE & $\begin{array}{c}\text { Nombre d'années d'exercice depuis sa } \\
\text { création }\end{array}$ & $\begin{array}{c}\text { Variable catégorielle : } \\
0 \mathrm{si}<=\text { à } 10 \text { ans } \\
1 \mathrm{si}>10 \text { ans }\end{array}$ \\
\hline CPT_HR & Disposer de RH compétentes & $\begin{array}{c}\text { Variable dichotomique : } \\
0 \text { si pas de RHC } \\
1 \text { si oui }\end{array}$ \\
\hline STR_V & Avoir une vision stratégique claire & $\begin{array}{c}\text { Variable dichotomique : } \\
0 \text { si pas de vision } \\
1 \text { si oui }\end{array}$ \\
\hline FIN_ACC & Avoir un accès facile au financement & $\begin{array}{c}\text { Variable dichotomique : } \\
0 \text { si accès difficile } \\
1 \text { si accès facile }\end{array}$ \\
\hline
\end{tabular}

Notre échantillon a été constitué sur la base des deux critères suivants :

- $\quad$ le CA annuel moyen $<200$ Mio MAD ;

- l'effectif permanent < 250 salariés pour s’aligner sur les critères de l'OCDE et de l'UE.

Au final, 174 PME répondant simultanément à ces critères ont été retenues pour les besoins de notre étude. Certains questionnaires ont été éliminés en raison de données manquantes ou de doublons.

Les caractéristiques de notre échantillon sont synthétisées dans le tableau 3 ci-après :

TABLEAU 3: Principales caractéristiques des PME de l'échantillon

\begin{tabular}{|c|c|c|c|}
\hline & & Frequency & Percent \\
\hline \multirow{4}{*}{ Régions $^{5}$} & Grand Nord & 33 & 19,0 \\
\hline & Centre & 117 & 67,2 \\
\hline & Grand Sud & 24 & 13,8 \\
\hline & Total & 174 & 100,0 \\
\hline \multirow{4}{*}{ Activity area ${ }^{6}$} & Industries lourdes (SEC1) & 39 & 22,4 \\
\hline & Distribution et services (SEC2) & 56 & 32,2 \\
\hline & Industries légères (SEC3) & 79 & 45,4 \\
\hline & Total & 174 & 100,0 \\
\hline \multirow{3}{*}{ Age of the SME } & $<=$ à 10 ans & 59 & 33,9 \\
\hline & $>10$ ans & 115 & 66,1 \\
\hline & Total & 174 & 100,0 \\
\hline
\end{tabular}

${ }^{5}$ Dans la région Grand Nord, nous avons compilé les régions administratives de TangerTétouan-Al Hoceima, de l'Oriental, de Fès-Meknès et de Rabat-Salé-Kénitra. Le centre comprend les régions de Béni Mellal-Khénifra et le Grand Casablanca-Settat. Et enfin, nous avons regroupé les régions de Marrakech-Safi, Drâa-Tafilalet, Souss-Massa, Guelmim-Oued Noun, Laayoune-Sakia El Hamra et Eddakhla-Oued Eddahab dans le Grand Sud.

${ }^{6}$ Les industries lourdes regroupent les secteurs Chimie-Parachimie, Electricité-Electronique et Mécanique-Métallurgie. Les industries légères comprennent les secteurs de l'Agroalimentaire, Textile-Cuir et Industrie Du Papier-Edition. 


\begin{tabular}{lccc}
\hline & Micro (0-9) & 22 & 12,6 \\
\cline { 2 - 4 } Number of & Small (10-49) & 82 & 47,1 \\
\cline { 2 - 4 } employees & Medium (50-249) & 70 & 40,2 \\
\cline { 2 - 4 } & Total & 174 & 100,0 \\
\hline
\end{tabular}

\section{Traitement des données}

Pour le traitement des données qualitatives, nous avons eu recours à une analyse de contenu, en procédant aux codages ouvert, axial et sélectif comme le suggèrent Strauss et Corbin (2004). Ainsi, à partir de la variable " indicateurs de mesure fréquemment utilisés », nous avons pu dégager 12 items chacun correspondant à un indicateur de mesure (voir GRAPHIQUE 1). Chacun de ces items a été ensuite codifié comme variable dichotomique (1 s'il est utilisé, 0 sinon).

La variable dépendante est également de nature dichotomique : avoir (ou pas) un SMP. Rappelons que celui-ci est défini par les indicateurs utilisés (quantitatifs et qualitatifs) et les objectifs poursuivis (pilotage et prise de décision). Ainsi, la valeur 1 est attribuée à la variable "SMP » pour toute PME qui utilise simultanément des indicateurs quantitatifs et qualitatifs pour le pilotage de la performance, et 0 sinon. De ce fait, en vue de relever les facteurs éventuels de contingence, nous nous sommes basés sur une régression logistique binaire.

Les variables indépendantes catégorielles ${ }^{7}$ intégrées dans cette régression sont récapitulées dans le tableau 4 suivant :

TABLEAU 4: Categorical Variables Codings

\begin{tabular}{|cc|c|c|}
\hline \multirow{2}{*}{ Having easy financing access } & Frequency & Parameter coding \\
\cline { 3 - 4 } & & & $(1)$ \\
\hline \multirow{2}{*}{ Having competent human ressources } & NO & 160 &, 000 \\
& YES & 14 & 1,000 \\
\multirow{2}{*}{ Having strategic vision } & NO & 130 &, 000 \\
& NO & 44 & 1,000 \\
& YES & 65 &, 000 \\
Age of the SME & $<=10$ years & 59 & 1,000 \\
& $>10$ years & 115 &, 000 \\
& & &
\end{tabular}

\section{Résultats et discussions}

\section{Indicateurs et SMP dans les PME enquêtées}

L'éventail des indicateurs de mesure utilisés dans les PME enquêtées reste limité, ne dépassant pas au total 12 comme le montrent les résultats du

\footnotetext{
${ }^{7}$ Ces variables ont été sélectionnées après un test de $\mathrm{Khi}^{2}$ significatif avec la variable «SMP».
} 
graphique 1 ci-dessous. Ce qui concorde avec les besoins simples des PME en matière d'information et de SMP comme le suggère la littérature.

GRAPHIQUE 1 : Classement des indicateurs de mesure par nature et fréquence d'utilisation

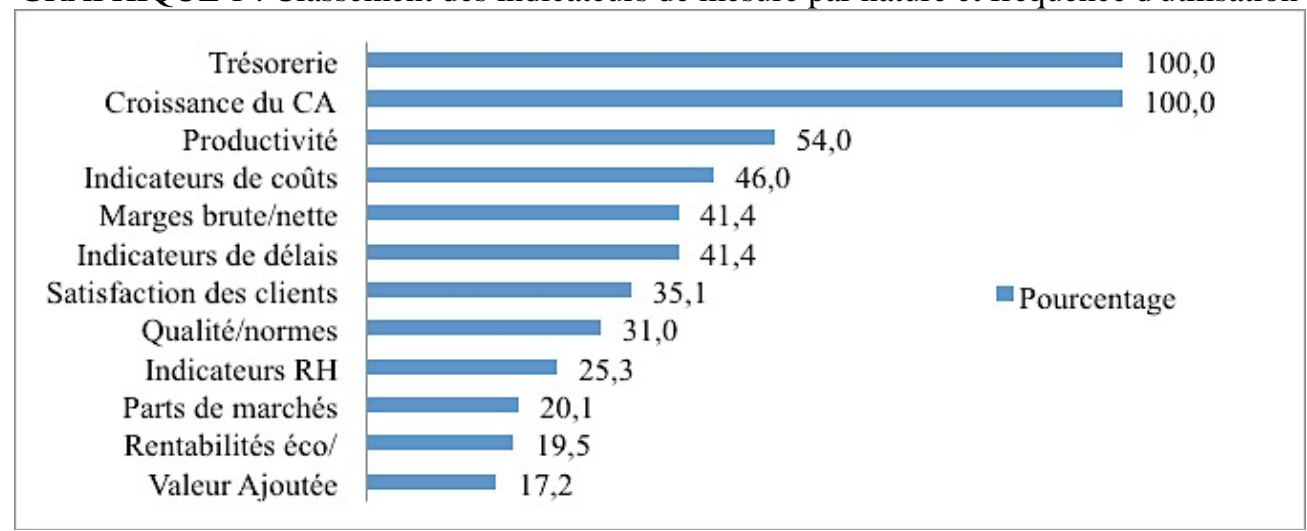

L'analyse de la nature de ces indicateurs montre le gap existant entre la littérature et la pratique des PME marocaines enquêtées. En effet, ces dernières utilisent aussi bien des indicateurs financiers que non financiers. Mieux encore, hormis le chiffre d'affaires et la trésorerie qui trônent à la première place, les indicateurs qualitatifs comme la productivité, la qualité ou la satisfaction des clients sont d'usage beaucoup plus fréquent que ceux liés à la rentabilité et à la valeur ajoutée.

En revanche, la mesure de la performance reste axée sur les aspects économiques et opérationnels notamment ceux rattachés à la production et aux clients. Ce qui corrobore le nombre limité de parties prenantes considérées par les PME.

Sur le plan financier, le suivi et la gestion de la trésorerie constituent le souci principal des PME beaucoup plus que celui de la rentabilité. En effet, tout besoin pèserait lourdement sur les charges financières, et partant, sur les résultats de l'entreprise mais aussi sur sa capacité d'autofinancement.

En outre, la majorité des PME se limite au suivi simultané de cinq indicateurs au maximum comme l'illustre le graphique 2 ci-après :

GRAPHIQUE 2: Poids des PME en fonction du nombre d'indicateurs utilisés simultanément

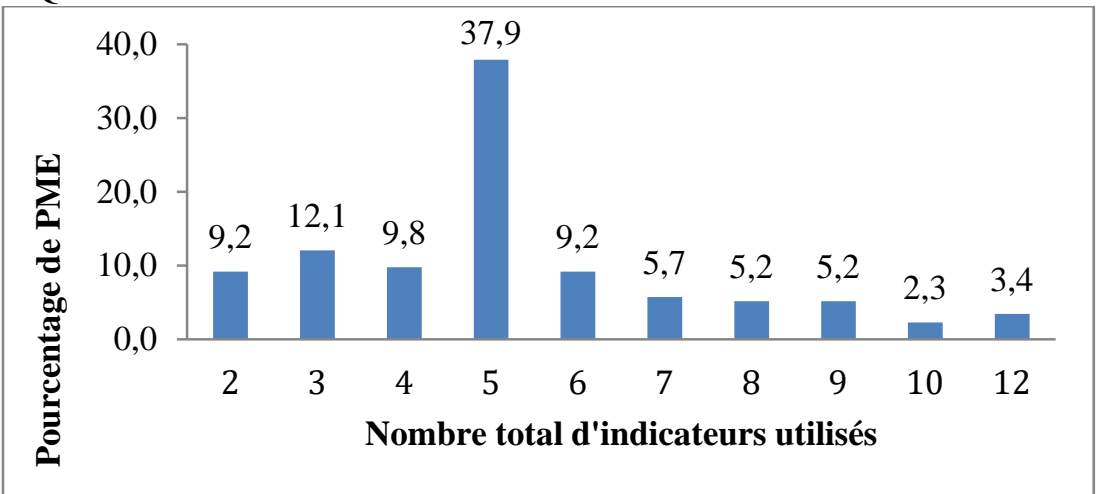


Cette parcimonie serait, selon Meyer $(2007)^{8}$, parmi les critères d'un SMP optimal ; car avec une pléthore d'indicateurs, les limites cognitives seraient dépassées et l'information perdue. Ce nombre limité d'indicateurs explique la faible formalisation des SMP au sein des PME marocaines sondées. En effet, seules 28\% des entreprises déclarent avoir un système de mesure structuré, le plus souvent sous forme de tableaux de bord périodiques. Les supports généralement utilisés sont des tableurs.

Quant aux objectifs de la mesure, si toutes les entreprises ont déclaré faire usage des indicateurs de mesure pour le contrôle et le suivi des axes de la performance qui les intéressent, seules 31\% affirment les utiliser aussi comme outils de pilotage de la performance de leur entreprise.

Toutefois, et en dépit des points soulevés, l'exploration des perspectives futures des PME montrent une évolution de leur perception du management et du pilotage de leur organisation vers une vision stratégique plus explicite, un besoin de renouvellement de leur style de management et une mise à niveau de leurs systèmes de mesure et de pilotage de la performance. En effet, plusieurs propriétaires-dirigeants ont exprimé leurs besoins d'accompagnement dans ce sens, comme en témoigne le graphique 3 ciaprès :

GRAPHIQUE 3 : Effectifs et fréquences des PME ayant exprimé des besoins d'accompagnement

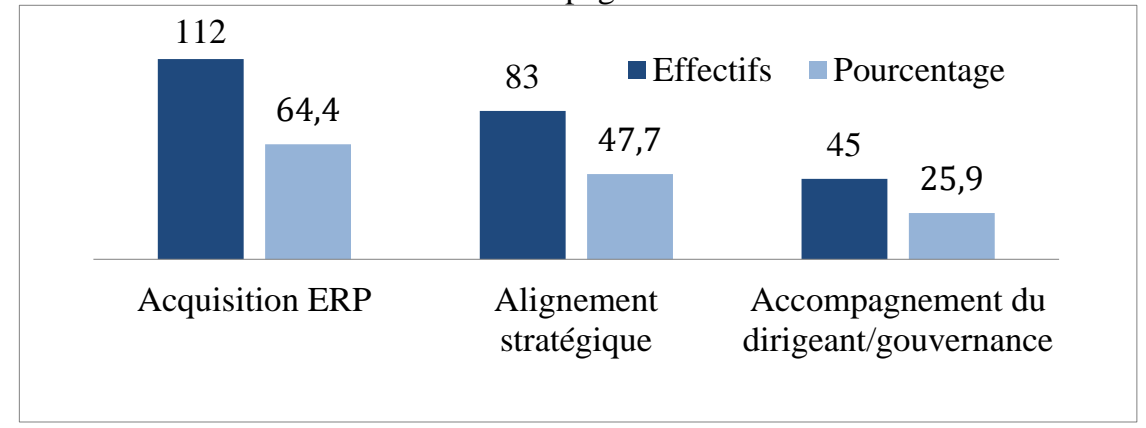

Ainsi, il apparait clairement que les systèmes d'information et de mesure de la performance "intégrés » constituent, désormais, une des préoccupations capitales des propriétaires-dirigeants. Conscients des mutations profondes de l'environnement liées à la mondialisation et aux progrès technologiques inextricables, ceux-ci considèrent que la mise en place de tels outils devient une nécessité. Les principales attentes exprimées sont notamment :

- $\quad$ le perfectionnement du système de calcul des coûts analytiques ;

\footnotetext{
${ }^{8}$ Il suffit selon l'auteur de peu d'indicateurs pour " garder une trace ». Il a même spécifié que trois indicateurs non financiers et trois autres financiers seraient largement suffisants.
} 
- $\quad$ l’amélioration du suivi de la productivité des ressources humaines et matérielles ;

- la rapidité d’obtention d’informations plus précises et donc une meilleure flexibilité ;

- $\quad$ la fluidité de circulation de l'information ;

- $\quad$ la synchronisation, en temps réel, entre les différentes fonctions de l'entreprise ; et plus particulièrement entre les fonctions approvisionnement, production, et commerciale. Donc, un gain énorme de temps et une réactivité accrue ;

- $\quad$ le suivi plus rigoureux des différentes opérations ;

- $\quad$ la prise de décision éclairée et rapide grâce à une meilleure visibilité des différents processus organisationnels.

Ceci résume l'évolution de la perception de ces systèmes, de simples outils de mesure et de reporting ex-post vers de véritables outils de pilotage et de management de la performance.

Pour une analyse plus fine, nous nous sommes interrogés sur l'existence d'éventuels facteurs de contingence à même d'influencer la structure et l'usage des SMP dans les PME.

\section{Les facteurs de contingence}

Comme signalé plus haut (2.2), l’étude de la relation entre la variable dépendante dichotomique «SMP » et les variables explicatives sélectionnées s'est faite à l'aide d'une régression logistique binaire. Les tests de spécification du modèle ainsi que les principaux résultats sont récapitulés dans les tableaux ci-dessous :

TABLEAUX 5a, 5b et $5 c$ : Tests de spécification du modèle

TABLEAU 5a: Omnibus Tests of Model Coefficients

\begin{tabular}{|cc|c|c|c|}
\hline & & Chi-square & df & Sig. \\
\hline \multirow{4}{*}{ Step 1 } & Step & 64,756 & 5 &, 000 \\
& Block & 64,756 & 5 &, 000 \\
& Model & 64,756 & 5 &, 000 \\
\hline
\end{tabular}

TABLEAU 5b: Model Summary

\begin{tabular}{|c|c|c|c|}
\hline Step & -2 Log likelihood & Cox \& Snell R Square & Nagelkerke R Square \\
\hline 1 & $140,217^{\mathrm{a}}$ &, 311 &, 449 \\
\hline
\end{tabular}

a. Estimation terminated at iteration number 5 because parameter estimates changed by less than ,001.

TABLEAU 5c : Hosmer and Lemeshow Test

\begin{tabular}{|c|c|c|c|}
\hline Step & Chi-square & df & Sig. \\
\hline 1 & 8,182 & 8 &, 416 \\
\hline
\end{tabular}

Les tests de spécification révèlent des seuils de signification probants. En témoigne également le tableau 6 de classification des prédictions : 
TABLEAU 6: Classification Table ${ }^{\mathrm{a}}$

\begin{tabular}{|c|c|c|c|c|c|}
\hline & \multirow{3}{*}{ Observed } & & \multicolumn{3}{|c|}{ Predicted } \\
\hline & & & \multicolumn{2}{|c|}{ Using a PMS } & \multirow[t]{2}{*}{ Percentage Correct } \\
\hline & & & $\mathrm{NO}$ & YES & \\
\hline \multirow{3}{*}{ Step 1} & \multirow{2}{*}{ Using a PMS } & $\mathrm{NO}$ & 115 & 11 & 91,3 \\
\hline & & YES & 23 & 25 & 52,1 \\
\hline & \multicolumn{2}{|c|}{ Overall Percentage } & & & 80,5 \\
\hline
\end{tabular}

a. The cut value is ,500

Les résultats définitifs de la regression logistique binaire sont repris dans le tableau 7 ci-après:

TABLEAU 7 : Variables in the Equation

\begin{tabular}{|rr|r|r|r|r|r|r|r|r|}
\hline & \multicolumn{1}{|c|}{ B } & S.E. & Wald & df & Sig. & Exp(B) & \multicolumn{2}{|c|}{$\begin{array}{c}\text { 95\% C.I.for } \\
\text { EXP(B) }\end{array}$} \\
\cline { 7 - 9 } & & & & & & & & & \\
& & & & & & & & Lower & Upper \\
\hline & AGE(1) &, 619 &, 479 & 1,670 & 1 &, 196 & 1,857 &, 726 & 4,747 \\
& C_HR(1) & 2,079 &, 455 & 20,904 & 1 &, 000 & 7,996 & 3,280 & 19,494 \\
Step 1 $^{\text {a }}$ & STR_V(1) & 1,371 &, 442 & 9,615 & 1 &, 002 & 3,939 & 1,656 & 9,369 \\
& FIN_ACC(1) & 2,021 &, 803 & 6,342 & 1 &, 012 & 7,546 & 1,565 & 36,375 \\
& SIZE &,- 004 &, 004 & 1,081 & 1 &, 299 &, 996 &, 988 & 1,004 \\
& Constant & $-2,642$ &, 511 & 26,718 & 1 &, 000 &, 071 & & \\
\hline
\end{tabular}

a. Variable(s) entered on step 1: AGE, C_HR, STR_V, FIN_ACC, and SIZE.

Il ressort de cette analyse que les variables les plus significatives sont, respectivement, les ressources humaines, la vision stratégique et l'accès au financement. Alors que la taille et l'âge ne semblent pas affecter de façon importante l'utilisation d'un SMP au sein des PMEs enquêtées.

En effet, d'après les résultats obtenus, les PMEs ayant déclaré disposer de ressources humaines compétentes ont une probabilité plus élevée d'avoir un SMP. De même que celles qui ont un accès plus facile au financement et une vision stratégique claire. Ce qui corrobore les résultats de la plupart des études menées dans ce sens. Ainsi, c'est le manque de ressources, tant humaines que financières, qui constitue l'obstacle majeur à l'adoption de SMP au sein des PMEs.

En revanche, la taille semble indiquer une relation inverse à l'usage des SMP : plus l'entreprise est de plus petite taille, et plus elle utiliserait un SMP. Cet argument peut être appuyé par la plus grande flexibilité des petites structures. Toutefois, le faible seuil de signification statistique ne permet pas de trancher en sa faveur. Il en est de même pour la variable "âge " qui penche vers une adoption des SMP par les PMEs ayant plus de dix années d'ancienneté. 


\section{Conclusion}

L'objet de cet article était principalement de questionner la problématique de la mesure de la performance dans les PMEs marocaines. L’intérêt pour cette thématique a été motivé par le manque flagrant d’études menées sur les SMP des PMEs dans un contexte de pays en développement.

Cette exploration a été réalisée grâce à une étude empirique auprès d'un échantillon de 174 PME marocaines.

Le rapprochement des résultats obtenus à la littérature en la matière a permis de soulever plusieurs points de convergence dont notamment :

- $\quad$ Le faible usage des SMP parmi les PMEs ;

- $\quad$ Les parties prenantes restent limitées essentiellement aux clients, aux concurrents et aux salariés ;

- $\quad$ Les SMP utilisés par les PME enquêtées demeurent majoritairement non formalisés, simples, avec peu d’indicateurs et orientés vers les aspects opérationnels ;

- $\quad$ L’alignement stratégique, même s’il est jugé important par la plupart des propriétaires-dirigeants, reste encore faible parmi les entreprises de notre échantillon.

- Le manque de ressources aussi bien humaines que financières constitue le principal frein à l'adoption de SMP au sein des PMEs.

En revanche, certains points de divergence sont également à soulever :

- $\quad$ Les indicateurs de mesure utilisés ne sont pas uniquement quantitatifs chez la majorité des PME enquêtées mais celles-ci s’intéressent davantage aux indicateurs qualitatifs ;

- $\quad$ Si les SMP sont principalement utilisés comme outils de suivi et de contrôle ex-post, ils tendent à devenir de véritables outils d’aide à la décision et de management de la performance. Nous avons noté également l'intérêt grandissant des PME pour les SMP « intégrés » de type ERP ;

- $\quad$ Le rôle de la taille et de l'âge comme facteurs de contingence n’a pas été statistiquement confirmé.

Ceci étant, et à l'instar de toute recherche empirique, certaines limites sont à prendre en considération. En effet, un biais de représentativité subsisterait toujours même si dans l'ensemble les tests de fiabilité menés se sont révélés significatifs.

La nature exploratoire de l'étude oblige également à restreindre l'étendue des aspects à analyser. Ainsi des questions importantes comme les ressources nécessaires à la mise en place des SMP, leur gestion, ou encore l'impact de leur usage (quand ils existent) sur la performance des PME n'ont pas pu être abordées. Ce qui laisse la voie ouverte à de nouvelles pistes de recherche. 


\section{References:}

1. Ajzen,M., RondeauX, G., Pichault, F. et TASKin, L. (2016). Performance et innovation en PME : une relation à questionner. Revue Internationale PME, 29(2), 65-94.

2. Акравот, S. ет KHAN, Z. (2015). Assessing the impact of performance measurement systems in northern Nigeria small businesses. European Scientific Journal, 11(4), 263-279.

3. Allali, B. (2003). Vision des dirigeants et internationalisation des PME marocaines et canadiennes du secteur agro-alimentaire. Thèse de doctorat en administration, Université de Montréal, école des HEC Montréal, Canada.

4. Andersen, H., COBbOlD, I. ET LAWRIE, G. (2001). Balanced scorecard implementation in SMEs: reflection in literature and practice. Papier publié à la SMESME Conference. Copenhagen, Danemark.

5. Ates, A., Garengo, P., CoccA, P. et Bititci, U. (2013). The development of SME managerial practice for effective performance management. Journal of Small Business and Enterprise Development, 20(1), 28-54.

6. Barnes, M., Dickinson, T., Coulton, L., Dransfield, S., Field, J., Fisher, N., SAUnders, I. ET SHAW, D. (1998). A new approach to performance measurement for small to medium enterprises. Dans Proceedings of the Performance Measurement - Theory and Practice Conference. Cambridge, Royaume-Uni.

7. Bergeron, H. (2000). Les indicateurs de performance en contexte PME, quel modèle appliquer? $21^{\text {ème }}$ congrès de L'AFC. Angers, France.

8. BiTITCI, U.S. (1995). Performance measurement for performance management. Papier présenté à la IFIP WG5.7 Working Conference. Seattle, USA.

9. Bititci, U.S., CARrie, A.S. et McDevitT, L. (1997). Integrated performance management systems: a development guide. International Journal of Operations \& Production Management, 17(5), 522-534.

10. Bititci, U.S., Turner, T. et Begemann, C. (2000). Dynamics of performance measurement Systems. International Journal of Operations \& Production Management, 20(6), 692-704.

11. Bititci, U.S., Garengo, P., Dorfler, V. et Nudurupati, S. (2012). Performance measurement: challenges for tomorrow. International Journal of Management Reviews, 14(3), 305-327.

12. Bourguignon, A. (1995). La performance, essais de définition. Revue Française de comptabilité, 269, 60-65. 
13. Bourguignon, A. (1997). Sous les pavés de la plage...ou les multiples fonctions du vocabulaire comptable: l'exemple de la performance. Comptabilité-Contrôle-Audit, 3, 89-101.

14. Brem, A., KREUSEL, N. ET NEUSSER, C. (2008). Performance measurement in SMEs: literature review and results from a German case study. International Journal of Globalisation and Small Business, 2(4), 411-427.

15. Charreire, S. et Durieux, F. (1999). Explorer et tester. Dans R.A. Thiétart et coll. (dir.), Méthodologie de Recherche en Management (p. 57-80). Paris, France, Dunod.

16. Chennell, A.F., Dransfield, S.B., Field, J.B., Fisher, N.I., SAUNDERS, I.W., ET SHAW, D.E. (2000). OPM ${ }^{\circledR}$ : A system for organisational performance Measurement. Presenté à Performance Measurement - Past, Present and Futur, University of Cambridge, Royaume-Uni.

17. Collins, O.F., MOORE, D.G. (1970). The Organization Makers: A Behavioral Study of Independent Entrepreneurs. New York: Appleton-Century-Crofts, Meredith Corp.

18. D’Amboise, G. (1996). La recherche en gestion des PME : des paradigmes en évolution. Cahier de recherche $n^{\circ} 96-11-08$ de la Chaire Maclean Hunter, HEC, Montréal.

19. FrANCO-SANTOS, M., KENNERLEY, M., MICHELI, P., MARTINEZ, V., MASON, S., MARR, B., GRAY, D. ET NEELY, A. (2007). Towards a definition of a business performance measurement system. International Journal of Operations \& Production Management, 27, 784-801.

20. Franco-SAntos, M., LUCIANETTI, L.ET BOURne, M. (2012). Contemporary performance measurement systems: A review of their consequences and a framework for research. Management Accounting Research, 23, 79-119.

21. Garengo, P. Biazzo, S. et Bititci, U.S. (2005). Performance measurement systems in SMEs: a review for a research agenda. International Journal of Management Reviews, 7(1), 25-47.

22. GARENGO, P. ET BITITCI, U.S. (2007). Towards a contingency approach to performance measurement: an empirical study in Scottish SMEs. International Journal of Operations and Production Management, 27, 802-825.

23. GHAlAyini, A.M., NOBLE, J.S. ET CROWE, T.J. (1997). An integrated dynamic performance measurement system for improving manufacturing competitiveness. International Journal of Production Economics, 48, 207-255. 
24. GuILhon, A. (1998). Vers une nouvelle définition de la PME à partir du concept de contrôlabilité. Dans O. Torrès (Coord.), PME : de nouvelles approches (p. 55-67). Recherches en gestion. Paris, Economica.

25. Hudson, M., Smart, P.A. And Bourne, M. (2001). Theory and practice in SME performance measurement systems. International Journal of Operations and Production Management, 21, 1096-1116.

26. Hvolby, H., AND ThORSTEnson, A. (2000), Performance measurement in small and medium-sized enterprises. Dans Proceedings $3^{\text {rd }}$ International Conference on Stimulating Manufacturing Excellence in SMEs, Coventry, Coventry University, 324-332.

27. JaOuen, A. (2010). Typologie de dirigeants de petite entreprise. Journal of Small business and Entrepreneurship, 23(1), 1-19.

28. JULIEN, P.-A. ET MARCHESNAY, M. (1987). La petite entreprise : principes d'économie et de gestion. Paris, Vuibert.

29. Julien, P.-A. et MARChesnay, M. (1996). L'entrepreneuriat. Paris, Economica.

30. Julien, P.-A. (1998). Stratégie et contrôle de l'information dans les PME. Pour un élargissement du concept d'entreprise afin de mieux appréhender la stratégie des PME dynamiques. Management International, 2(2), 51-59.

31. KAPlAn, R.S. ET NoRTON, D.P. (1992). The balanced scorecard measures that drive performance. Harvard Business Review, JanvierFévrier, 71-90.

32. KAPlAn, R.S. ET NoRTON, D.P. (1996). The Balanced Scorecard Translating Strategy into Action. Harvard Business School Press, Boston, MA.

33. Kotey, B. et Meredith, G.G. (1997). Relationships among owner/manager personal values, business strategies and enterprise performance. Journal of Small Business Management, 35(2), 37-61.

34. LAITINEN, E.K. (2002). A dynamic performance measurement system: evidence from small Finnish technology companies. Scandinavian Journal of Management, 18, 65-99.

35. LANGWERDEN, E.F. (2015). Performance Measurement System development in SMEs: testing and refining the circular methodology. $5^{\text {th }}$ IBA Bachelor Thesis Conference. Enschede, The Netherlands.

36. LAufER, J.C. (1974). Comment on devient entrepreneur? Revue française de gestion, 2, 18- 29. 
37. LeBAS, M. (1995). Performance measurement and performance management. International Journal of Production Economics, 41, 2335.

38. LYNCH, R.L. ET CROSS, K.F. (1991). Measuring up-The essential guide to measuring business performance, Mandarin, London.

39. MELnYK, S., BITITCI, U.S, PlATtS, K., TOBIAS, J. ET ANDERSEN, B. (2014). Is performance measurement and management fit for the future? Management Accounting Research, 25(2), 173-186.

40. MEYER M.W. (2002). Rethinking performance measurement: beyond BSC. Cambridge University Press, New York.

41. MEYeR M.W. (2007). Finding performance: The new discipline in management. Dans Business Performance Measurement, Cambridge University Press, Second edition, pp. 51-61.

42. MC MAHON, R.G.P. (2001). Deriving an empirical development taxonomy for manufacturing SMEs using data from Australia's business longitudinal survey. Small Business Economics, 17(3), 197212.

43. Miles, M.B. et Huberman, M.A. (2003). Analyse des données qualitatives (2e éd.). Bruxelles : Édition de Boeck.

44. Miles, R. ET SNOW, C. (1978). Organizational strategy, structure and process. New York: McGraw Hill.

45. NeELy, A. (1999). The performance measurement revolution: why now and what next? International Journal Of Operations \& Production Management, 19(2), 205-228.

46. Neely, A., AdAms, C. AND CROWE, P. (2001). The performance prism in practice. Measuring Business Excellence, 5(2), 6-12.

47. Neely, A., Mills, J., Platts, K., Richards, H., Gregory, M., Bourne, M. ET Kennerley, M. (2000). Performance measurement system design: developing and testing a process-based approach. International Journal of Operations \& Production Management, 20(10), 1119-1145.

48. NUDURUPATI, S.S., BITITCI U.S., KUMAR V. ET CHAN F.T.S. (2011). State of the art literature review on performance measurement. Computers \& Industrial Engineering, 60(2), 279-290.

49. OCDE, (2004). Partenariats, Grappes, Réseaux et Droits de Propriété Intellectuelle : Perspectives et Enjeux des PME Innovantes dans une Économie Mondialisée. $2^{\text {ème }}$ conférence de l'OCDE des Ministres en charge des PME. Istanbul, Turquie.

50. ORIOT, F. ET BERGERON, H. (2012). Indicateurs de performance et priorités stratégiques des dirigeants de PME. Dans G.Lecointre (dir.), Le grand livre de l'économie PME (p. 201-225). Gualino Lextenso Editions. 
51. Russell, R. (1992). The role of performance measurement in manufacturing excellence. Papier présenté à la BPICS Conference, Birmingham, UK.

52. St-PIERRE, J. EL FADIL, J. ET PILAEVA, K. (2012). Les objectifs des entrepreneurs et leur conception de la performance : une étude comparative de dirigeants canadiens et marocains. Dans Business and entrepreneurship in Africa, BEA PROCEEDINGS Volume $n^{\circ} 1$ (p.94125). Québec City, Québec, Canada.

53. ST-PiERre, J. ET CADIEUX, L. (2011). La conception de la performance: quels liens avec le profil entrepreneurial des propriétaires dirigeants de PME ? Revue de l'Entrepreneuriat, 10(1), 33-52.

54. SALGADO, M. (2013). La performance : une dimension fondamentale pour l'évaluation des entreprises et des organisations. <hal00842219>.

55. Shahbaz, M.A., Javed, A., Dar, A. et SATtar, T. (2014). Performance measurement of small and medium enterprises (SMEs) in Pakistan. Archives of Business Research, 2(3), 42-49.

56. SMith, N. R. (1967). The entrepreneur and his firm: The relationship between type of man and type of company. East Lansing: Bureau of Business and Economic Research, Graduate School of Business Administration, Michigan State University.

57. STRAUSS, A. L., ET CORBIN, J. (2004). Les fondements de la recherche qualitative : techniques et procédures de développement de la théorie. Fribourg, Suisse : Academic Press Fribourg.

58. TAticchi, P., TONElli, F. And CAgnazzO, L. (2010). Performance measurement and management: a literature review and a research agenda. Measuring Business Excellence, 14(1), 4-18.

59. TORRÈs, O. (1999). Les PME. Paris, Flammarion, coll. Dominos.

60. TORRÈs, O. (2008). Comprendre le management des PME : le rôle clé de la proximité. Conférence à l'EM. Lyon.

61. Vygotsky, L.S. (1978). Mind in society: the development of higher psychological processes. Cambridge, MA: Harvard University Press.

62. Welsh, J.A ET WHITE, J.F. (1981). A small business is not a little big business. Harvard Business Review, 59(4), 18-32.

63. White, B., BEnNeTt, R. ET SHIPSEY, R. (1982). Information and the Small Manufacturing Firm. Report of a study of information use and needs in Small Manufacturing firms in UK and the current pattern of information position, funded by the British Library Research and Development Department. Edinburgh, UK. 